\title{
AUTONOMOUS NAVIGATION SYSTEM BASED ON GPS
}

\author{
Zhaoxiang Liu, Gang Liu ${ }^{*}$ \\ Key Laboratory of Modern Precision Agriculture System Integration Research, China \\ Agricultural University, Beijing, China, 100083 \\ * Corresponding author, Address: P.O. Box 125, China Agricultural University, Qinghua \\ Donglu 17, Haidian District, Beijing, 100083, P. R. China, Tel: +86-10-62736741, Fax: +86- \\ 10-62736746, Email: pac@cau.edu.cn
}

\begin{abstract}
An autonomous navigation system based on GPS was developed. The system was composed of the under-controlling part and the decision-making part; the two parts communicated with each other via wireless data transmission modules. The under-controlling part included the ARM7 microprocessor, the wireless data transmission module, the GPS receiver and the mobile quadricycle. The decision-making part included the laptop and the wireless data transmission module, the path planning algorithm based on Visual C++ 6.0 and MapObjects. The autonomous navigation and long-distance control of the quadricycle were realized. To evaluate the performance of the system, some tests were performed.
\end{abstract}

Keywords: GPS, autonomous navigation, wireless data transmission, MapObjects

\section{INTRODUCTION}

The development emphasis of agricultural engineering is artificial intelligence control and agricultural mechanical automatic system in the $21^{\text {th }}$ century. The application of agricultural intelligent technology will promote automatization of the agricultural mechanism and the automatization of the agricultural mechanism will play an important role in the digital agricultural production system in the future (Jiang Chen, 2005).

With the development of agricultural productivity, the agricultural tractor becomes more and more good-sized, and the farmers more and more rely on 
several high-power tractors in the agricultural production. So it is necessary to maximize the efficiency of the tractors. The autonomous navigation technology of agricultural vehicle can realize high-efficiency fieldwork without interference of human beings for a long time, and improve the accuracy of operation at the same time. What is more, it can also reduce the cost of production, improve the quality of farm produce and relieve the working load of drivers.

As a positioning sensor, GPS has been widely used in autonomous navigation system of vehicle for which to supply high-accuracy positioning information. The application of autonomous navigation technology based on GPS in agricultural vehicle can realize the consistency of operation, improve the performance of the agricultural mechanism, realize working at night, prolong the working time, relieve the working intensity of drivers and make the drivers spend more attention on the status of farming apparatus which can improve the working quality (Defeng Kong, 2007). So it is significant to study on the autonomous navigation technology of agricultural vehicle.

The research target of the autonomous navigation system based on GPS is to make the vehicle have the ability of autonomous positioning and path planning, to realize autonomous driving. In this study, a mobile quadricycle was used as the test vehicle.

\section{DESIGN OF SYSTEM}

Autonomous navigation system based on GPS was consisted of undercontrolling part and decision-making part (Figure 1).

Satellite positioning information

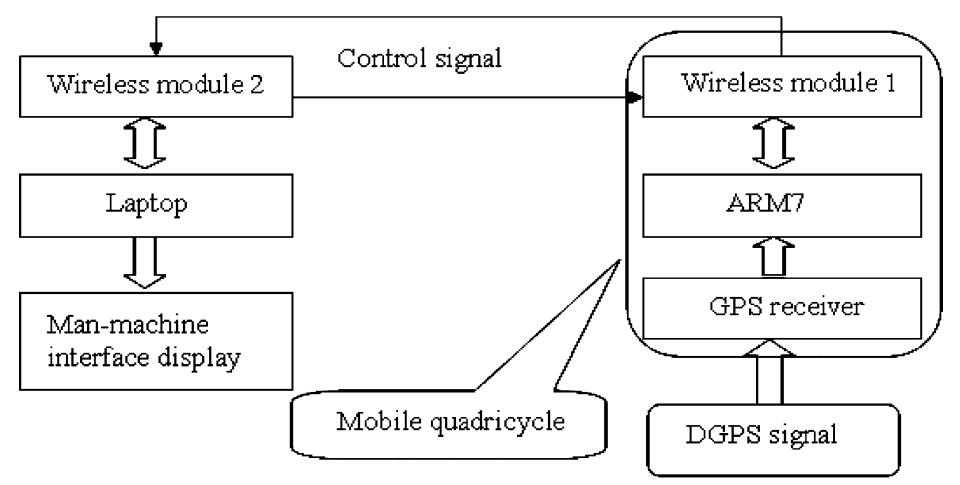

Figure 1. General structure of the system

The under-controlling part included a wireless data transmission module, an ARM7 microprocessor, a GPS receiver and a mobile quadricycle. The decision-making part was composed of a wireless data transmission module and a laptop. The two parts communicated with each other via the wireless 
data transmission modules. The under-controlling part received the GPS signal via GPS receiver, and transmitted the GPS data to the decisionmaking part via the wireless data transmission module 1 . The decisionmaking part received the GPS information via wireless data transmission module 2. After processing and analyzing in the laptop, the decision-making part transmitted the control information to the under-controlling part, which controlled the mobile quadricycle to run, turn left or right and stop. And the position of the quadricycle was displayed by the man-machine interface at real-time.

\section{IMPLEMENTATION OF SYSTEM}

\subsection{The under-controlling part}

Figure 2 showed the structure of the under-controlling part.

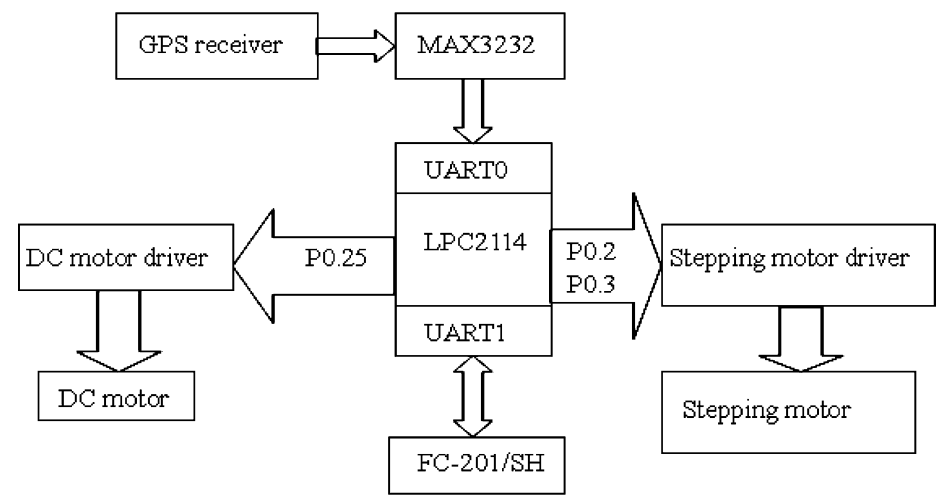

Figure 2. Structure of the under-controlling part

The under-controlling part was made up of ARM7 microprocessor (LPC 2114), wireless data transmission module (FC-201/SH), GPS receiver (AgGPS 132) and mobile quadricycle. The LPC 2114 was used as control unit, its UART0 which connected with the GPS receiver by the level translator of MAX3232 chip was used to receive the GPS data, and its UART1 which connected with the FC-201/SH directly was used to transmit the GPS data to the decision-making part and receive the control information from the decision-making part. The mobile quadricycle assembled a DC motor, which was used to drive the quadricycle, a DC motor driver, two steeping motors, which were used to control the swerve of the quadricycle and two steeping motor drivers. The I/O port P0.25 of LPC 2114 connected with the DC motor driver, I/O port P0.2 and P0.3 connected with the stepping motor drivers. 
The software of under-controlling part included the main program initiating the system and waiting interrupt, the interrupt 0 subprogram receiving and transmitting the GPS data, and the interrupt 1 subprogram receiving control information and controlling the action of quadricycle.

\subsection{The decision-making part}

Figure 3 showed the structure of the decision-making part.

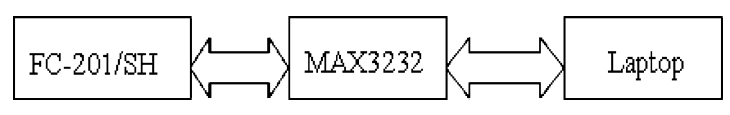

Figure 3. Structure of the decision-making part

The decision-making part included laptop and wireless data transmission module (FC-201/SH). They connected with each other via the level translator of MAX3232 chip. The FC-201/SH was used to receive the GPS data and transmit the control information, the laptop was used to analyze and process the data, make the controlling decision and display the position of the quadricycle at real-time.

The software of decision-making part was developed based on Visual C++ 6.0 and MapObjects. It included ten parts, which were described as follows:

(1) The main program: its task was to set the navigation path, receive the GPS data from the under-controlling part, convert the coordinates and control the status of the quadricycle.

(2) The setting serial port subprogram: it was implemented by adopting Microsoft Communications Control (MSComm), its main task was to choose serial port and set the baud rate and so on.

(3) The collecting the data of navigation path subprogram: its task was to receive the GGA sentences of NMEA-0183 format from a moving GPS receiver along the navigation path, and use the CStdioFile class of Microsoft to save the data in a text file.

(4) The setting navigation path subprogram: the CFile class and CArchive class were adopted to obtain the GGA sentence from the text file, then convert it into Gauss Plane Coordinates, and then save the coordinates in the CArray class, finally, display it in form of point on the map.

(5) The receiving GPS data subprogram: it obtained a GGA sentence by judging two marking bits of ' $\$$ ' and '*' in the GGA sentence via serial port.

(6) The extracting longitude and latitude from the GGA sentence and converting coordinates subprogram.

(7) The displaying position subprogram: its main task was to project the point onto the map according to the coordinates realizing displaying the 
position of the quadricycle. It was realized by adopting dynamic tracking layer of MapObjects.

(8) The controlling status with straight navigation path subprogram: its main task was to make a controlling decision by comprehending the position information of quadricycle.

(9) The path transformation of complicated path subprogram: its task was to transform the complicated path into many short straight paths, which were used to match the complicated path.

(10) The manual control subprogram: in order to avoid damaging the quadricycle when autonomous navigation was out of control, manual control program was designed to control the quadricycle to run, turn left, turn right and stop by pressing the control button manually.

\subsection{Navigation algorithm}

Considering the accuracy of the GPS receiver was not very high and the speed of the quadricycle was slow in actual application, the following algorithm was chosen (Figure 4).

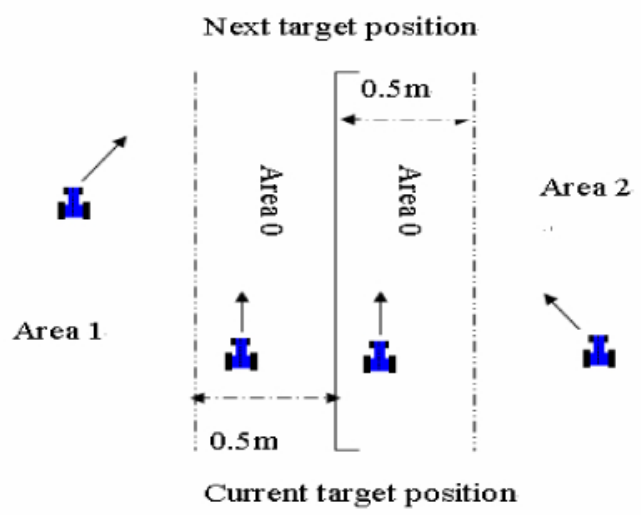

Figure 4. Sketch map of the navigation algorithm

The width of the area 0 was set as $1 \mathrm{~m}$, if the quadricycle was in the area 0 , it was instructed to continue running, if in the area 1, it was instructed to turn right, if in the area 2, it was instructed to turn left. This kind of algorithm had higher efficiency because the frequent turning was not needed. The algorithm was described as follows:

It was supposed that the real-time position of the quadricycle was $\left(X_{0}, Y_{0}\right)$, the current target position was $\left(X_{1}, Y_{1}\right)$, the next target position was $\left(X_{2}, Y_{2}\right)$ and the equation of the straight path, which was formed by the current target position and the next target position, was formulated as:

$$
A X+B Y+C=0
$$


The vertical distance from the real-time position to the straight path formed by the current target position and the next target position was used to determine whether the quadricycle needed turning, the orientation relationship between the real-time position and the straight path was used to determine the direction of the turning.

\section{PERFORMANCE TEST}

\subsection{Experiment equipment}

The experiment equipment for performance test included a mobile quadricycle, a DGPS (AgGPS 132), which included a base station and a mobile station, an IBM T43 laptop (1.86GHz CPU, 512M RAM) and a software developed based on Visual $\mathrm{C}++6.0$ and MapObjects. Figure 5 showed the experiment equipment.

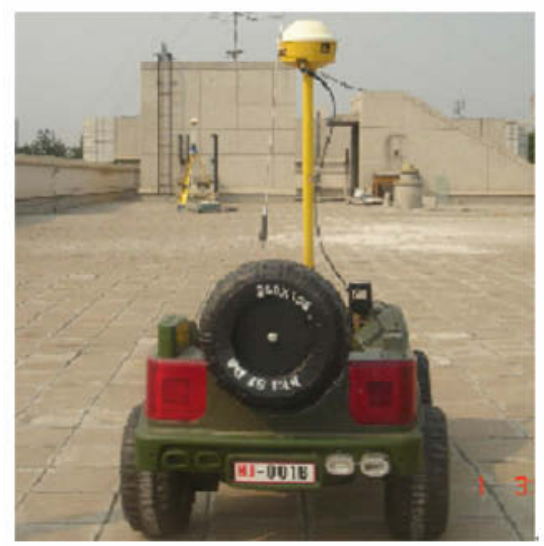

Figure 5. The experiment equipment

\subsection{Result and discussion}

Tests were performed on the top of the Computer Network Center building in China Agriculture University. Before the tests, real-time data was collected by walking along a straight path $(20 \mathrm{~m})$ and a rectangular path $(20 \mathrm{~m} \times 11 \mathrm{~m})$, and then saved as desired paths. The tracking accuracy was measured using the lateral deviation of the quadricycle center of gravity (CG) from a reference line, namely the desired path (Q. Zhang, 2004).

In the navigation test with straight path, the quadricycle was placed with a 1.5-meter deviation from the path to the left at the beginning of the path, and 


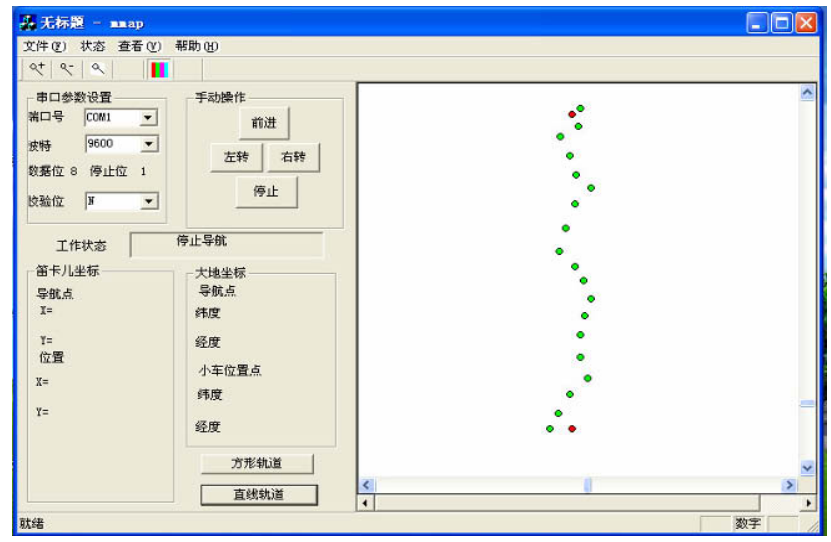

Figure 6. Navigation result with straight path

the speed of the quadricycle was $1.1 \mathrm{~m} / \mathrm{s}$. Figure 6 showed the navigation result with straight path.

As shown in Figure 6, two red points formed the straight path, and the green points represented the real-time positions of the quadricycle, the maximum lateral deviation was less than $0.71 \mathrm{~m}$.

In the navigation test with rectangular path, the quadricycle was also placed with a 1.5-meter deviation from the path to the left at the beginning of the path, the speed of the quadricycle near the four corners was about 0.8 $\mathrm{m} / \mathrm{s}$, and the rest time was $1.1 \mathrm{~m} / \mathrm{s}$. Figure 7 showed the navigation result with straight path.

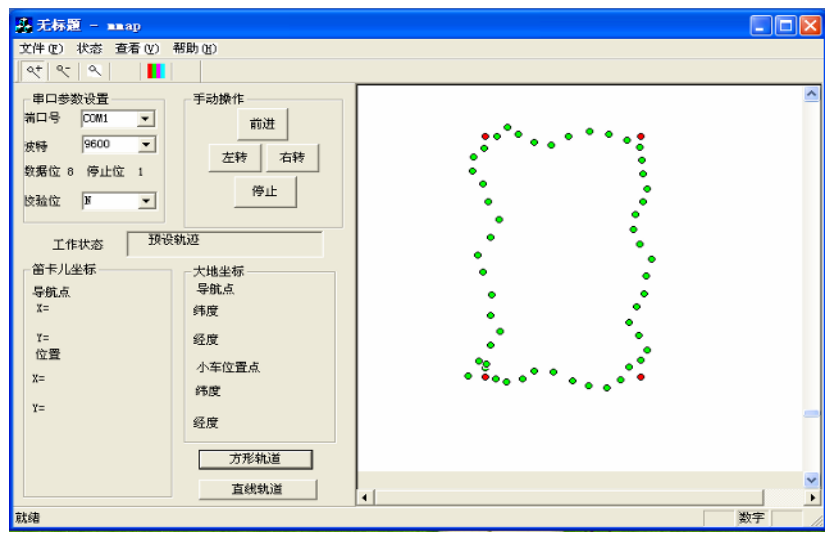

Figure 7. Navigation result with rectangular path

As shown in Figure 7, four red points formed the rectangular path, and the green points represented the real-time positions of the quadricycle. The density of green points at the corners was higher than others, because the speed at the corners was slower than others, the maximum lateral deviation was less than $0.82 \mathrm{~m}$ at the corners and others were less than $0.74 \mathrm{~m}$. 


\section{CONCLUSIONS}

An autonomous navigation system based on GPS was developed. The following conclusions were inferred from the study:

(1) Hardware platform of autonomous navigation system was developped based on ARM7 microprocessor. Dynamic collection of GPS data on the quadricycle and dynamic control of the quadricycle was realized.

(2) Control software of autonomous navigation system in the decisionmaking part was developped based on Visual C++ 6.0 and MapObjects. Autonomous driving of the quadricycle was realized and the real-time position of the quadricycle could be displayed by the control software.

(3) Long-distance control of the quadricycle was realized by adopting the wireless data transmission modules.

(4) Tests were performed on the top of the Computer Network Center building in China Agriculture University. When tracking the straight path, the maximum lateral deviation was less than $0.71 \mathrm{~m}$, and when tracking the rectangular path, the maximum lateral deviation was less than $0.82 \mathrm{~m}$ at the corners and others were less than $0.74 \mathrm{~m}$. According to the results, the navigation algorithm could fulfill the task effectively.

(5) In order to improve the accuracy and efficiency of the autonomous navigation system, the following research should be emphasized on increasing the navigation sensor, adopting multi-sensor fusion technology and improving the navigation algorithm.

\section{ACKNOWLEDGEMENTS}

This paper is supported by the national 863 projects: Control Technique and Product Development of Intelligent Navigation of Farming Machines (2006AA10A304).

\section{REFERENCES}

Defeng Kong, Chun Wang, Xi Wang, Nan Jiang, Design of Large Tractor Automatic Guidance System Based on GPS/GIS, Journal of Agricultural Mechanization Research, No. 3, 2007, pp. 54-55 (in Chinese).

Jiang Chen, Shuaibing Shi, Juncai Hou, Jie Xu, The Development of Digital Agriculture and Chinese Agriculture Machinery, Journal of Agricultural Mechanization Research, No. 4, 2005, pp. 21-23 (in Chinese).

Q. Zhang, H. Qiu, A Dynamic Path Search Algorithm for Tractor Automatic Navigation, American Society of Agricultural Engineers, Vol. 47, No. 2, 2004, pp. 639-646. 